\section{Avertissement au lecteur}

Cet article est une approche critique des tests de traction utilisés pour évaluer la solidité et la résistance des arbres Statics Integrated Assessment (évaluation intégrée de la statique) et Static integrated Method, SIA (méthode statique intégrée). Nous avons décidé, avec l'assentiment de l'auteur que nous remercions, de publier la traduction intégrale de cet article paru dans ARB Magazin (titres, sous-titres et illustrations sont toutefois de la rédaction). Il répond à certaines interrogations de lecteurs de LA FORÊT et de WALD und HOLZ sur la thématique des tests de traction mécaniques et des modèles qui leur servent de base.

Avec l'afflux de promeneurs et de visiteurs en forêt et la multiplication des installations (bancs, places de jeux ...) se pose pour les propriétaires et forestiers

la question de leur responsabilité vis-à-vis de ces usagers, d'autant plus encore dans les forêts des zones urbaines ou périurbaines. Il est bon, pensons-nous, que les forestiers et les propriétaires disposent d'informations sur les méthodes de diagnostic de sécurité des arbres et qu'elles ne soient pas exclusivement réservées aux spécialistes de la question, arboristes, spécialistes des soins aux arbres, entreprises prestataires de services, etc. C'est d'autant plus important que les forestiers sont de plus en plus appelés à entretenir des arbres et des zones boisées enclavées dans des zones d'habitation ou les jouxtant immédiatement.

\footnotetext{
* Peter Sterken est expert en arboriculture. Ce scientifique possède un bureau de conseil indépendant en Espagne. L'article original en anglais «Tree risk assessment: a review of methods», est paru dans ARB Magazin, 180 Spring Issue 2018, pp. 40-43, The Arboricultural Association.

Traduction: Ferdinand Oberer
}

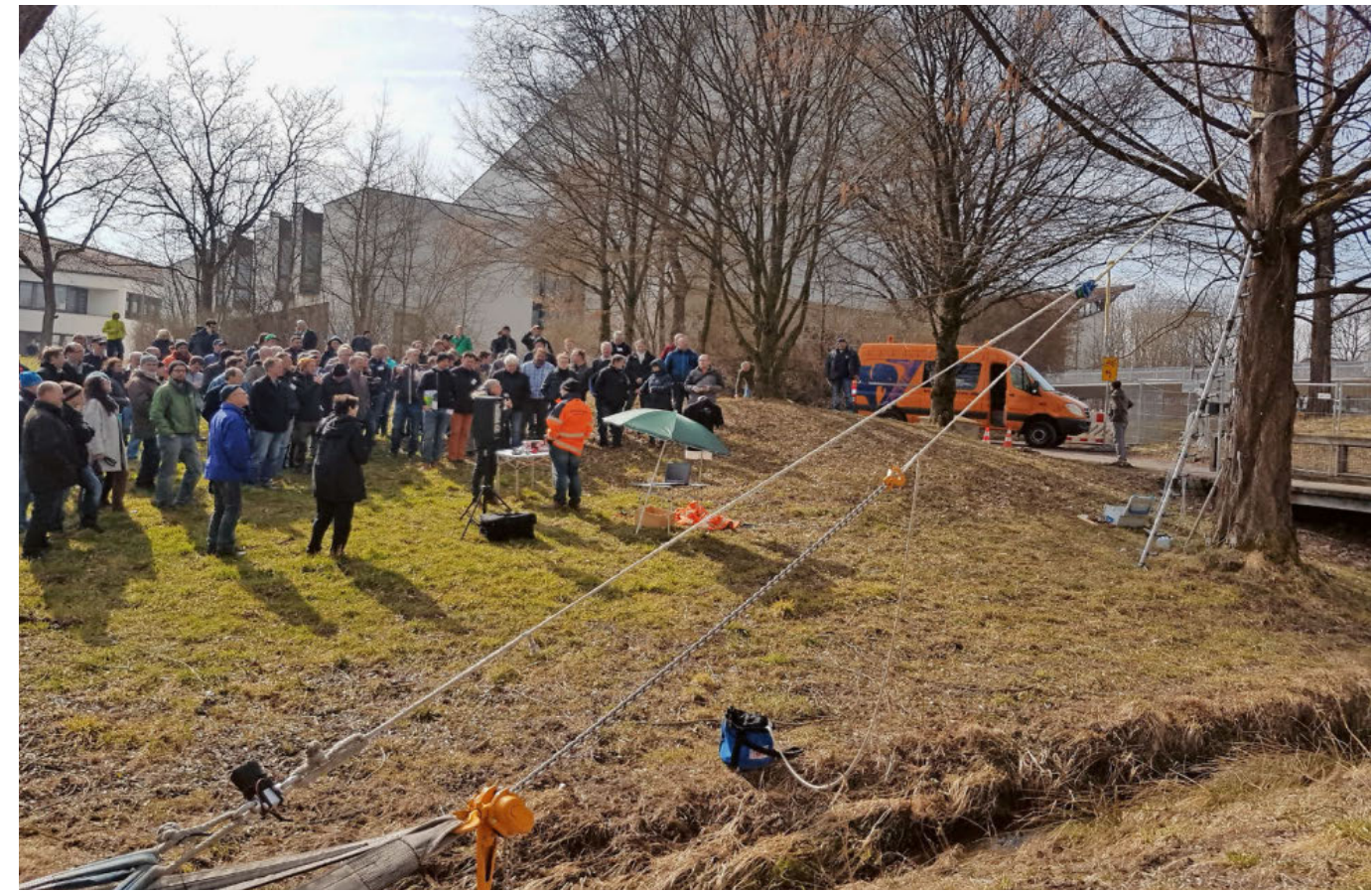

Test de traction en marge du Forum bavarois de l'arbre, en mars dernier à Freising (D).

Ferdinand Oberer

\title{
Les bases scientifiques des tests de traction
}

\section{Peter Sterken* | Trouver une méthode fiable et précise pour évaluer la dangerosité de l'arbre est le Graal de l'arboriculture urbaine depuis des décennies. Les conséquences étant lourdes en cas d'erreur, les arboristes doivent remettre en question les méthodes à leur disposition.}

Une méthode d'évaluation visuelle «combinée» de la dangerosité de l'arbre a été publiée par Sterken (2006): la méthode $\mathrm{V}$ calcule la vitesse de vent critique à laquelle l'arbre est susceptible de rompre. La méthode SIA (Statics Integrated Assessment, Wessoly and Erb, 1998) calcule le point hypothétique de rupture ainsi qu'un coefficient de sécurité globale lorsque l'arbre est soumis à une certaine vitesse de vent. Lors des tests de traction dits SIM (Statics Integrated Methods, Wessolly and Erb, 1998), les arbres sont soumis à des contraintes statiques afin de calculer leur coefficient de sécurité hypothétique (le point de rupture ou de déracinement de l'arbre). Dans le sillage de ces méthodes, d'autres approches ont été développées.

Le présent article propose une mise à jour des limites de ces méthodes d'évaluation; en effet, si on fait aveuglément confiance à l'une ou l'autre, on s'expose à un risque de défaillance inattendu.

\section{La théorie face aux réalités de terrain}

Il n'existe encore aucune méthode précise pour prévoir la défaillance d'un arbre (James et al., 2014) et aucune d'elles ne peut revendiquer une garantie contre la rupture, le déracinement ou les défaillances du houppier. Cette réalité doit être mise dans la balance, face aux coûts souvent élevés de méthodes de diagnostic à l'aide d'instruments.

En outre, la preuve scientifique n'est pas faite que ces méthodes sont fondées sur des mesures d'effets réels de vents agissant sur des arbres réels. «Il est reconnu qu'il n'existe pas encore de preuves statistiques démontrant un rapport entre les résultats pronostiqués par le modèle et les résultats réels» (Sterken, 2006).

De même, les arbres peuvent rompre ou se déraciner sous des charges de vent bien en deçà des seuils indiqués par les tests de traction. Autrement dit, un arbre peut céder contre toute attente 
après avoir été diagnostiqué «sûr». A ce sujet, des résultats importants ont été publiés par Baker and Bell (1992) et reproduits par James (2010). Petola (2006) remarque aussi que les processus sous-jacents aux dégâts provoqués par le vent ne sont pas encore entièrement compris. Par conséquent, ces méthodes ne peuvent qu'indiquer une probabilité de défaillance.

L'évaluation de la résistance mécanique à l'aide d'une simulation des charges statiques (tests de traction) seule n'est donc pas suffisante, et le calcul de la force du vent agissant sur le houppier est problématique lorsque les profils aérodynamiques de l'arbre sont inconnus (Petola et al., 2006). Le fendage du bois au niveau des contreforts des racines, dû à la force du vent ou aux tests de traction (Claire et al., 2003), devrait également être pris en compte car beaucoup d'arbres cèdent de cette façon.

\section{Méthode inutile?}

Certains mécanismes récurrents de défaillances structurelles et la complexité des charges ne peuvent pas toujours être pronostiqués par les méthodes V, SIA, SIM ou similaires (Sterken, 2005 et 2006). Selon Wessolly et Erb (2006, page 233), le mécanisme de rupture d'un arbre creux cadre avec le flambage d'un brin d'herbe. Partant de cette hypothèse, ses auteurs «autovalident» la valeur informative de leur méthode de tests de traction. Ceci devrait susciter de sérieux doutes auprès de toute personne ayant l'expérience de la grande variété de mécanismes de rupture des arbres creux. De surcroît, l'herbe n'a rien à voir avec le bois.

De plus, Wessoly et Erb écrivent que «dans des zones où des défaillances structurelles se manifestent plus tôt, on devrait vérifier de près si les cavités régulières

\section{LA MÉTHODE «ELASTO-INCLINO»}

Des tests de traction sont effectués pour déterminer à la fois la résistance d'un arbre contre la rupture et contre le déracinement [méthode «elasto-inclino»].

Les élastomètres [capteurs en haut sur l'image, appelés aussi jauges de déformation] enregistrent les déformations des fibres du bois. Les capteurs à la base du tronc sont des inclinomètres qui captent des inclinaisons au niveau du collet avec une précision d'un centième de millimètre. L'ancrage d'un arbre est calculé selon la «courbe de basculement générale» qui décrit le processus du déracinement des arbres selon les indications des auteurs.

Examen d'un arbre, avec les différents capteurs en place. $\quad$ Ferdinand Oberer

s'étendent sur une grande longueur à l'intérieur de l'arbre [sic]». Dans la réalité, beaucoup d'arbres endommagés présentent des cavités irrégulières. Cela signifie-t-il que le raisonnement exposé dans Wessolly et Erb (2006) n'est donc pas applicable à l'examen des arbres présentant des cavités irrégulières? Laisser de côté ces arbres réduirait considérablement l'efficacité et l'utilité d'une telle méthode d'évaluation.

Les structures mécaniques des arbres sont complexes (en particulier celles des sujets manifestement défaillants, candidats typiques des tests de traction selon les méthodes SIA et autres); elles ne peuvent pas être considérées comme des poutres par-

\section{LE TEST DE TRACTION}

Le test de traction SIA [Static Integrated Assessment] ou SIM [Statics Integrated Method] est une méthode d'évaluation approfondie lorsque l'arbre présente des signes de défaillances mécaniques visibles. Il a été introduit dans les années 1980 en tant que méthode nondestructive. Lors du test de traction, l'arbre est soumis à une charge dosée à l'aide d'un câble dans la direction présumée du vent dominant. Sous cette charge, les fibres du bois dans le tronc se déforment en deçà de leur limite d'élasticité, et les déformations sont relevées par des élastomètres. Sur la base de ces mesures et à l'aide de paramètres [diamètre du tronc, hauteur de l'arbre, forme du houppier], un ordinateur branché aux élastomètres calcule si l'arbre résiste à une poussée du vent de force 12 sur l'échelle de Beaufort ou pas. D'autres variables d'entrée pour les calculs sont le coefficient de traînée de l'essence en présence, ainsi que son coefficient de charge admise, c'est-à-dire les propriétés mécaniques des bois verts, empruntés aux tableaux du «Catalogue de résistance des bois verts de Stuttgart». Les calculs se font selon les modèles de flambage d’une coque cylindrique.

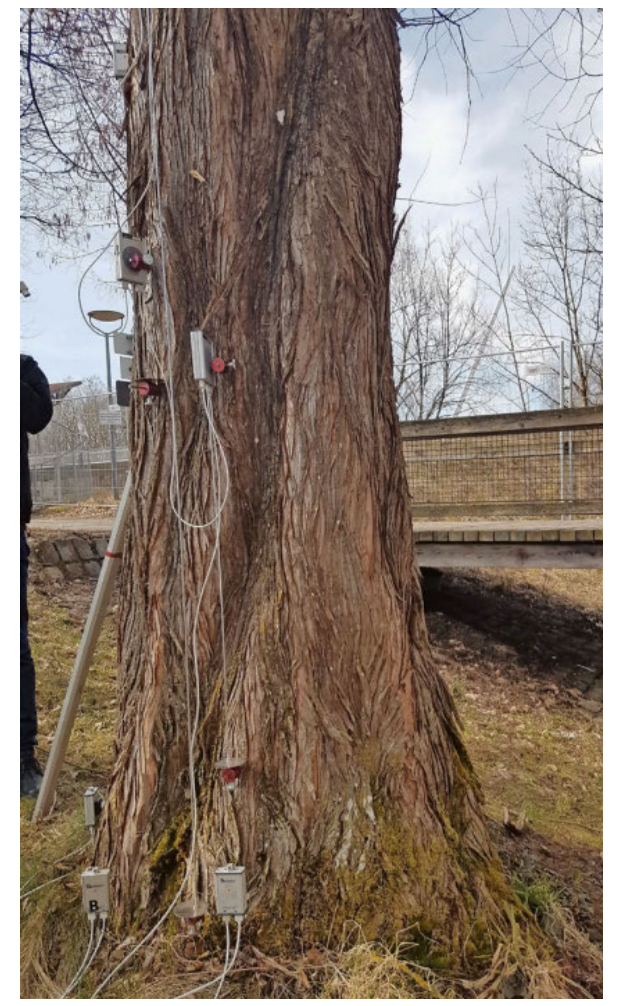

faites. Or, les méthodes d'évaluation décrites dans le présent article présupposent que l'arbre correspond à une poutre parfaite, ou à un tube constitué d'un matériau isotrope (matériau ayant les mêmes propriétés physiques dans toutes les directions). En outre, la poussée du vent à laquelle l'arbre est supposé céder est calculée suivant la théorie de la limite d'élasticité des fibres (du bois) sous compression axiale.

Le lecteur devrait se poser les questions suivantes (notamment par rapport au raisonnement de Wessolly et Erb, 2006, page 233, discuté ci-dessus): «Les arbres que je dois évaluer correspondent-ils vraiment à des poutres ou des tubes parfaits? Ne présentent-ils pas plutôt des cavités excentriques, des fissures, des géométries irrégulières, des troncs courbés, etc.?» La réponse à la première question sera très probablement «non» et celle à la deuxième «oui», car si ces arbres n'étaient pas sévèrement endommagés, ils n'auraient pas besoin d'examen à l'aide des méthodes mentionnées. L'utilité de ces dernières doit donc être mise en question.

\section{Absence de preuves scientifiques}

La prudence s'impose pour d'autres raisons encore: le socle de la méthode proposée par Wessolly et Erb (2006) - notamment les coefficients de traînée, les propriétés mécaniques des bois selon le «Catalogue de 
résistance des bois verts de Stuttgart» (voir encadré «Test de traction») et la courbe de basculement générale (voir encadré «Méthode elasto-inclino») - devrait reposer sur des données scientifiquement démontrées. Or nous n'avons pas trouvé dans l'ouvrage de Wessolly et Erb (2006) de références à des publications scientifiques, examinées par des pairs, qui reposeraient sur des données et des procédures scientifiquement reconnues. Par conséquent, on ne peut que conjecturer: les coefficients de traînée, les propriétés mécaniques des bois et la courbe de basculement générale sont-ils ou non purement hypothétiques? Sans même compter que les propriétés mécaniques des bois de Niklas et Spatz (2010) pourraient remplacer le «Catalogue de résistance des bois verts de Stuttgart» (Wessolly et Erb, 1998), puisqu'elles sont plus exhaustives et qu'elles ont été publiées dans une revue soumise à évaluation par des pairs.

A cela s'ajoute que les troncs et les branches courbes peuvent se fissurer dans le sens longitudinal avant qu'une compression des fibres du bois se produise (Ennos et van Casteren, 2009), ce qui signifie que toutes les trois méthodes comparées dans le présent article (même les tests de traction à l'aide des élastomètres) sont inutiles en présence de ce type de défaillances très courant, car elles ne prennent en compte que la compression des fibres du bois (Wessolly et Erb, 2016).

Les méthodes mentionnées n'utilisent d'ailleurs qu'une seule valeur de module d'élasticité et une valeur de densité par espèce d'arbre. Quant à la densité, Spatz et Pfister (2013) préconisent un coefficient de sécurité de $200 \%$, au lieu des $150 \%$ des tests de traction SIM. En accord avec Sterken (2006), ils écrivent que «les arbres réels et les vents réels ne s'adaptent pas aux modèles mathématiques» et que les arbres forment des structures dynamiques.

Mon intention n'est pas d'invalider les méthodes mentionnées ci-dessus, mais plutôt de démontrer qu'elles n'en sont encore qu'aux prémices. L'interprétation correcte de leurs résultats peut sauver des vies; c'est la raison de cet article, étant entendu que le modèle V (Sterken 2006) souffre des mêmes limitations.

\section{Epaisseur de paroi résiduelle «représentative»}

Mattheck et al. (2008) ont critiqué la méthode SIA, les tests de traction (SIM) et les sections transversales «résistantes aux tempêtes» de Lothar Wessolly. Ils ont ignoré une source importante qui corrobore leurs dires: Detter et al. (2005) affirment qu'«ils (les tests de traction à l'aide d'élasto- et d'inclinomètres) permettent également de déterminer les épaisseurs de paroi résiduelles sans recourir à des outils invasifs.» Pour illustrer ces propos, un diagramme des épaisseurs de paroi résiduelles de 4807 arbres censément examinés figure dans leur document; ceci suggère qu'un grand nombre d'arbres subsistent avec des très petits coefficients $t / R$ (épaisseur de paroi résiduelle/diamètre du tronc). Ce même diagramme a été reproduit par Bond (2006). Wessolly et Erb (1998) en ont publié une version antérieure.

Mais ces résultats devraient être interprétés avec précaution car ils peuvent être trompeurs; en effet, les dimensions des cavités, telles que publiées par Detter et al. (2005), sont basées sur quatre types de variables d'entrée:

a) sur les modules d'élasticité hypothétiques empruntés au «Catalogue de résistance des bois verts de Stuttgart» (Wessolly et Erb 2016);

b) sur des calculs des profils reposant sur des mesures au compas et supposant, sans que ce soit certifié, que ces profils épousent des formes elliptiques;

c) sur la mesure des extensions des fibres de bois à l'aide d'élastomètres, sous l'effet d'une charge mécanique statique;

d) sur des formules d'ingénieur empruntées à des manuels de base.

Examinons chacun de ces types de variables:

a) les propriétés des matériaux, dont le mode d'élasticité, peuvent varier au sein d'une espèce, voire d'un profil transversal d'un arbre ou de ses différentes parties. Les valeurs peuvent donc diverger des données des tableaux utilisées pour les tests de traction;

b) beaucoup d'arbres présentent un profil transversal irrégulier, non elliptique, à la base de leur tronc et au niveau des empattements. La méthode «élastomètre» fait appel au compas pour mesurer les plus grands diamètres à la hauteur de l'emplacement des élastomètres (à l'endroit présumé du défaut mécanique ou de la cavité), puis les calculs qui en découlent reposent sur un profil transversal elliptique. Dès lors, les calculs se font à partir de profils transversaux beaucoup plus importants que dans la réalité, en raison des irrégularités des profils consécutives à la présence de contreforts de racines, etc. De telles surestimations de la géométrie porteuse amènent à une vision faussée de la rigi-

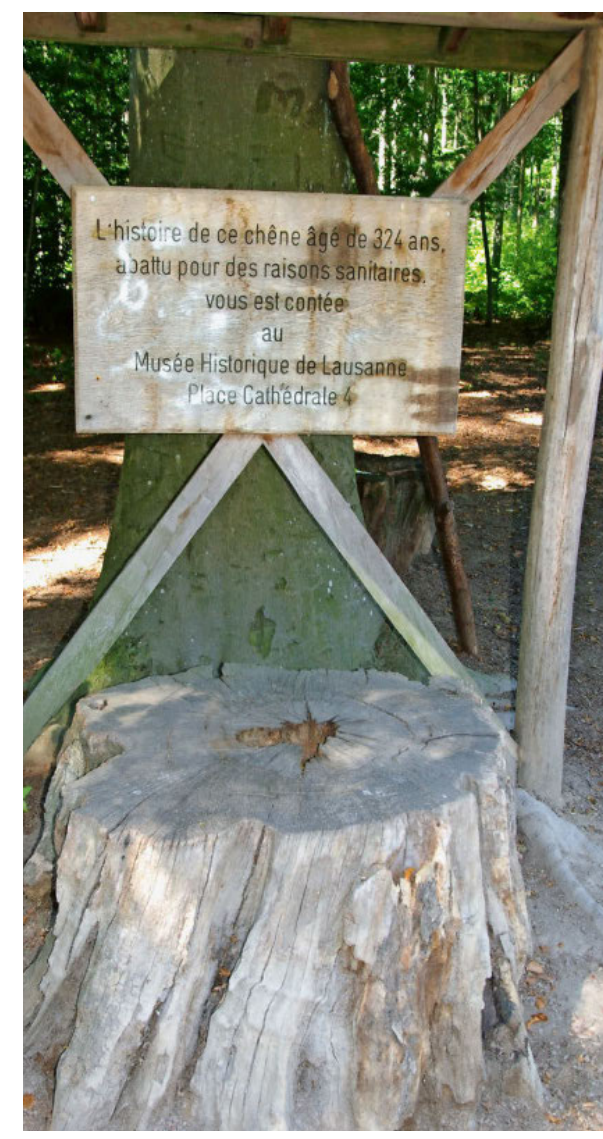

En zones habitées et périurbaines et en présence de public, le rapport à l'arbre change, ce qui induit de nouvelles exigences en termes de sécurité et de responsabilité des propriétaires et des forestiers. Alain Douard

dité et de la résistance à la flexion de la zone évaluée;

c) l'importance de l'extension des fibres de bois, lorsque l'arbre est soumis aux efforts du test de traction, est mesurée à l'aide des élastomètres. Si ces jauges de déformation ne sont pas positionnées correctement, par exemple sur une zone bombée du tronc ou sur un contrefort de racine, les valeurs mesurées peuvent être plus importantes que les déformations microscopiques réelles, ce qui constitue une source d'erreur supplémentaire;

d) finalement, des formules d'ingénieur empruntées à des manuels de base sont appliquées à des valeurs issues des profils transversaux surestimés, à des modules d'élasticité hypothétiques (qui peuvent facilement être plus élevés que dans la réalité) et à des déformations mesurées sous l'effort des tests de traction (dont la valeur relevée peut être plus élevée que les déformations réelles). 
En résumé, les résultats des calculs peuvent indiquer une rigidité bien plus faible que celle établie par le test de traction; ensuite, l'épaisseur de paroi résiduelle est simplement calculée sur la base des tests de traction à l'aide des formules empruntées aux manuels; enfin, comme toutes les hypothèses peuvent être mises en doute (sauf celle de l'extension des fibres, pour autant que les élastomètres aient été correctement positionnés), le résultat peut également être mis en question. Et c'est pourquoi les calculs des coefficients de cavité tels que publiés par Detter et al. (2005), Bond et Wessolly et Erb devraient être pris avec précaution.

\section{Hypothèses non vérifiées}

Les épaisseurs de paroi résiduelles, telles que publiées dans Detter et al. (2005), ne sont d'ailleurs pas des valeurs réelles mais des valeurs hypothétiques. Interpréter ces chiffres comme des valeurs réelles serait une erreur dangereuse. Il nous paraît osé de prétendre que «2171 arbres sur pied présentent des coefficient $t / R$ entre 0,0 et $0,3 »$ : dans Wessolly et Erb (2016), nous n'avons pas trouvé de références renvoyant à des publications scientifiques examinées par des pairs, qui apporteraient des données fiables et qui s'appuyeraient sur des méthodes scientifiquement reconnues. Elles seules pourraient conforter cette affirmation.

Si les arbres soumis à des efforts de traction avaient été coupés après les tests pour relever le coefficient $t / R$ (à condition que leurs parois résiduelles correspondassent à des cercles concentrés et fermés), et si ces relevés eussent été comparés aux coefficients hypothétiques respectifs, alors il existerait une preuve corroborant cette affirmation ambitieuse. Mais dans la bibliographie de Wessolly et Erb (2016), on ne trouve aucune référence à des sources qui renseigneraient sur les méthodes et les formules appliquées.

Dans Wessolly et Erb (2016), le même diagramme revient, cette fois avec les résultats de tests de traction sur 8140 arbres. Il suggère que 3729 arbres sur pied présentent un coefficient $t / R$ entre 0,0 et 0,3 . Si les auteurs admettent cette fois que ce diagramme est le résultat de calculs issus des relevés d'élastomètres, ils ne fournissent aucune explication ni avertissement à ce propos. Wessolly et Erb seraient donc bien avisés (aussi bien que les autres auteurs ayant publié cette idée) de revoir leur affirmation et leur diagramme qui conduisent à des interprétations erronées. Un public de non-experts pourrait facilement être induit en erreur et faussement conclure que les arbres avec des coefficients $t / R$ proches de 0,0 peuvent être considérés comme sûrs.

\section{Discussions autour du coefficient $\mathbf{t} / \mathbf{R}$}

Enfin, Gruber (2008) affirme que la règle $\mathrm{t} / \mathrm{R}=0,32$ de la méthode VTA (Visual Tree Assessment) ainsi que le diagramme renommé de Mattheck seraient erronés. Selon lui, aussi bien le diagramme de Mattheck que celui de Wessolly et Erb devraient être remis en doute d'un point de vue scientifique. Les contrôles de sécurité des arbres reposent de longue date sur ces deux documents.

Une dispute acharnée sur les coefficients $\mathrm{t} / \mathrm{R}$ agite la profession des arboristes et des soins aux arbres depuis les années 1990. Les discussions tournent toujours autour de l'épaisseur de la paroi résiduelle nécessaire à la sécurité de l'arbre en présumant une paroi résiduelle régulière et fermée. Mais combien d'arbres creux correspondent réellement à ce rêve d'ingénieur, c'est-àdire à des tubes réguliers constitués d'un matériau isotrope? Ils sont rarissimes. La plupart des cavités sont excentriques, irrégulières, ouvertes ou liées à d'autres faiblesses structurelles comme des fissures, des entre-écorces, des nœuds, etc. Face à la présence de telles éléments, ces discussions autour du $\mathrm{t} / \mathrm{R}$ paraissent ridicules.

\section{Valeurs précieuses pour s'orienter}

Huang et al. (2017) recommandent des valeurs de coefficients $t / R$ critiques, basées sur un flambage «Brazier», précédé d'une ovalisation du tronc lorsque l'arbre est soumis à des contraintes de flexion. Ils indiquent les coefficients $t / R$ suivants en fonction des modes de défaillance:

- pour un flambage «Brazier» $(0<\mathrm{t} / \mathrm{R}<0,6)$; - pour un cloquage tangentiel suivi par un fendage longitudinal $(0,06<t / R<0,27)$;

- pour un flambage «conventionnel» sous un effort de flexion $(0,27<t / R<1)$.

Ces résultats pourraient fournir des orientations précieuses pour évaluer des arbres creux. Les méthodes décrites ci-dessus ne seraient dès lors utilisables et crédibles qu'en présence de coefficients $t / R$ égaux ou supérieurs à 0,27 . Un coefficient $t / R$ inférieur à 0,27 provoquerait une défaillance mécanique du tronc creux qui serait imprévisible par les méthodes décrites ci-dessus; ceci donne plus de crédibilité à la fameuse valeur de 0,32 de Mattheck, valeur provenant d'une ancienne publication de Wagener (1963), selon l'affirmation de Gruber (2008).

\section{Recommandations}

Sur la base des discussions qui précèdent, on propose les orientations qui suivent. Elles pourraient servir dans l'attente d'une méthode de contrôle de sécurité plus fiable que celles disponibles aujourd'hui. Si un arbre creux présente une paroi résiduelle tubiforme et sans défaut mécanique (ce qui est l'exception), la valeur limite de 0,27 paraît justifiable pour éviter une défaillance selon les mécanismes mentionnés ci-dessus. Un tel chiffre devrait correspondre à un coefficient de sécurité de $200 \%$ par rapport à la charge du vent. Ce facteur peut se calculer en s'aidant de la formule $7 \mathrm{de}$ Sterken (2006).

Si le coefficient $t / R$ de la paroi résiduelle se situe en deçà de 0,27 , l'arbre entre dans le royaume des défaillances imprévisibles. On devrait évidemment tenir compte des problèmes mentionnés ci-dessus ainsi que de la complexité des forces, des propriétés des matériaux et de l'architecture de l'arbre. Seuls entrent en ligne de compte des contrôles visuels, aucune méthode assistée par ordinateur n'étant capable de s'y substituer. L'œil exercé de l'expert reste donc l'instrument crucial pour ce type d'examen.

Des recherches sur les palmiers fortement endommagés pourraient être conduites en plaçant sur leurs tiges des jauges de déformation de telle manière qu'elles puissent mesurer les déformations des fibres du bois dans toutes les directions anatomiques, lorsque ces palmiers sont soumis à une force de traction. On peut imaginer que c'est une combinaison des contraintes (p. ex. contrainte tangentielle, cisaillement, torsion) appliquées à une faible résistance du bois contre le fendage qui déclenche un effondrement de l'arbre, plutôt qu'une contrainte unidirectionnelle dans le sens longitudinal du tronc et parallèle aux fibres (Sterken, 2005, p. 42, et Clair et al., 2003).

Des tailles de réduction et des haubanages peuvent être envisagés pour éviter l'effondrement d'un arbre, au cas où des morceaux de tronc et des branches sont faiblement fixés. Certains affirment que des haubans synthétiques favorisent (comme Wessolly et Erb, 2016) la compensation des défaillances en permettant des mouvements légèrement atténués grâce à des amortisseurs. Ces affirmations n'ont pas encore été prouvées par des publications scientifiques reconnues. Elles demeurent donc des hypothèses. 